\author{
Wojciech Broniowski · Enrique Ruiz Arriola
}

\title{
Valence Double Parton Distributions of the Nucleon in a Simple Model
}

Received: 31 October 2013 / Accepted: 30 January 2014 / Published online: 15 February 2014

(C) The Author(s) 2014. This article is published with open access at Springerlink.com

\begin{abstract}
Valence double parton distribution functions of the nucleon are evaluated in the framework of a simple model, where the conservation of the longitudinal momentum is taken into account. The leading-order DGLAP QCD evolution from the low quark-model scale to higher renormalization scales is carried out via the Mellin moments of the distributions. Results of the valence quark correlation function show that in general the double distributions cannot be approximated as a product of the single-particle distributions.

Theoretical interest in double parton distributions (dPDFs) in hadrons has been recently renewed (see the reviews $[1,2]$ and the references therein). The activity is largely triggered by the experimental program at the LHC, where the double parton scattering (DPS) may bring substantial contribution in certain production processes [3,4]. Phenomenological aspects of the inclusion of the multi-parton effects have been widely pursued since the early days of the parton model [5], while many formal features of the multiparton evolution [6,7] follow from a pioneering study on fragmentation functions [8]. Elements of the QCD formulation are presented in [9-12], while $\mathrm{a}_{2}$ GPD interpretation is provided in [13]. Positivity bounds for dPDFs were recently discussed in [14].

Dynamic modeling of dPDFs has been up to now very little explored. In fact, the only calculations which nonperturbatively predict the valence dPDFs of the nucleon have been carried out in the framework of the MIT bag model [15] and in the constituent quark model [16]. In both calculations, the approximate implementation of relativity has the side effect of producing distributions with unphysical support, i.e., extending outside the interval $0 \leq x_{1}, x_{2}, x_{1}+x_{2} \leq 1$. An important aspect of the dPDFs is that they allow to detect tight diquark correlations, in which case the dPDF becomes a function of the sum $x_{1}+x_{2}$. In the $\left(x_{1}, x_{2}\right)$ distribution plot the fingerprints are the straight-line structures inclined by $45^{\circ}$.

The purpose of this talk is to explore the valence dPDF of the proton in a very simple model which includes the exact momentum-conservation constraints and complies to the Lorentz invariance. Satisfaction of these requirements results in proper theoretical features, such as the correct support in the $x$-variables or the quarknumber and momentum sum rules [17]. In the considered model, the correlations between partons are due
\end{abstract}

Talk presended by WB at the Light Cone 2013 Conference, Skiathos, Greece. Supported by Polish Ministry of Science and Higher Education (grant DEC-2011/01/B/ST2/03915), and by Spanish DGI (Grant FIS2011-24149) and Junta de Andalucía (Grant FQM225).

W. Broniowski $(\varangle)$

The H. Niewodniczański Institute of Nuclear Physics, Polish Academy of Sciences,

31342 Kraków Poland

E-mail: Wojciech.Broniowski@ifj.edu.pl

W. Broniowski

Jan Kochanowski University, 25406 Kielce, Poland

E. Ruiz Arriola

Departamento de Física Atómica, Molecular y Nuclear and Instituto Carlos I de Física

Teórica y Computacional Universidad de Granada, 18071 Granada, Spain 
solely to the longitudinal momentum conservation. Importantly, we carry out the LO DGLAP evolution [6,7] of the obtained valence dPDF, confirming its crucial effects for the corresponding quark correlation function as the evolution scale is moved up to the experimentally-accessible values. We find that with the increasing renormalization scale, the correlation between the valence quarks becomes large and positive along the edges where $x_{1}$ or $x_{2}$ is small, while it is negative in the region where $x_{1}$ and $x_{2}$ are similar and relatively large. This shows in explicit terms that approximating dPDFs with a product of two single-parton distributions (sPDF) is not justified.

Similarly to SPDF, which is interpreted as the probability distribution for the probed parton to carry the fraction $x$ of the total $p^{+}$momentum of the hadron, the dPDF has the interpretation of the joint probabilistic distribution that the $j_{1}$ and $j_{2}$ partons carry the momentum fractions $x_{1}$ and $x_{2}$ of the hadron, respectively. Denoting the sPDF as $D_{j}(x)$ and dPDF as $D_{j_{1} j_{2}}\left(x_{!}, x_{2}\right)$, one may define the parton correlation function $[15,16]$

$$
\rho_{j_{1} j_{2}}\left(x_{1}, x_{2}\right)=\frac{D_{j_{1} j_{2}}\left(x_{1}, x_{2}\right)}{D_{j_{1}}\left(x_{1}\right) D_{j_{2}}\left(x_{2}\right)}-1 \text {. }
$$

As mentioned, a dynamical nonperturbative calculation for the proton has been made in the MIT bag model [15], where the correlations stem from the conservation of the linear momentum (implemented in terms of the PeierlsYoccoz projection). As is well known, projecting onto good linear momentum coincides with boosting from the rest frame only for the exact eigenstates of relativistic systems [18]. A constituent quark model study has been carried out in [16]. Here we consider a much simpler model which in our view grasps the essential features of the problem and has the proper support. The role of transverse degrees of freedom is discussed at the end.

Let us introduce the probability that the parton carries the momentum fraction $x$ as the square of its wave function, $\phi(x)=|\psi(x)|^{2}$. Then the three-particle probability distribution is proposed in the form

$$
D_{3}\left(x_{1}, x_{2}, x_{3}\right)=\phi\left(x_{1}\right) \phi\left(x_{2}\right) \phi\left(x_{3}\right) \delta\left(1-x_{1}-x_{2}-x_{3}\right),
$$

where the delta function enforces the longitudinal momentum conservation. Its marginal projections define the $\mathrm{dPDF}$ and SPDF:

$$
\begin{aligned}
D_{2}\left(x_{1}, x_{2}\right) & =\int_{0}^{1} d x_{3} D_{3}\left(x_{1}, x_{2}, x_{3}\right)=\phi\left(x_{1}\right) \phi\left(x_{2}\right) \phi\left(1-x_{1}-x_{2}\right), \\
D_{1}\left(x_{1}\right) & =\int_{0}^{1} d x_{2} D_{2}\left(x_{1}, x_{2}\right)=\int_{0}^{1} \theta\left(1-x_{1}-x_{2}\right) d x_{2} \phi\left(x_{1}\right) \phi\left(x_{2}\right) \phi\left(1-x_{1}-x_{2}\right) .
\end{aligned}
$$

Note that Eq. (4) is equivalent to the quark number sum rule of Gaunt and Stirling (GS) [17]. The momentum GS sum rule is also satisfied, as it is straightforward to show that

$$
\int_{0}^{1-x_{1}} d x_{2} x_{2} D_{2}\left(x_{1}, x_{2}\right)+\int_{0}^{1-x_{1}} d x_{3} x_{3} D_{2}\left(x_{1}, x_{3}\right)=\left(1-x_{1}\right) D_{1}\left(x_{1}\right) \text {. }
$$

As a matter of fact, the only feature used in these derivations is the symmetric form of Eq. (2). In addition, due to the symmetry, the extremum of $D_{3}\left(x_{1}, x_{2}, x_{3}\right)$ is located at the symmetric point $x_{1}=x_{2}=x_{3}=1 / 3$ regardless of its particular shape.

Early multiparton models [5] take the simple Regge-motivated parametrization $\psi(x) \sim x^{a}$ with $a=1-$ $\alpha(0)$ and the intercept $\alpha(0)=1 / 2$, while the constant phase-space model of Ref. [19] uses $\psi(x)=1$. In this presentation we investigate two simple classes of models of the form

$$
\phi(x) \equiv|\psi(x)|^{2}=A x^{\alpha}(\text { model I }), \quad \phi(x) \equiv|\psi(x)|^{2}=A(1-x)^{a}(\text { model II) },
$$

where $A$ is a normalization constant such that $\int_{0}^{1} d x \phi(x)=1$. We consider the cases $\alpha=0,1 / 2,1$ and $a=1,2$. The first class corresponds to the so-called valon model $[20,21]$ for the proton, which assumes 
$G_{\text {uиd } / p}\left(x_{1}, x_{2}, x_{3}\right)=\left(x_{1} x_{2}\right)^{\alpha} x_{3}^{\beta} \delta\left(1-x_{1}-x_{2}-x_{3}\right)$ for $\alpha=\beta$. For instance, for the case of the second class with $a=2$ in Eq. (6) we get

$$
\begin{aligned}
D_{2}\left(x_{1}, x_{2}\right) & =\frac{1008}{29}\left(1-x_{1}\right)^{2}\left(1-x_{2}\right)^{2}\left(x_{1}+x_{2}\right)^{2}, \\
D_{1}(x) & =\frac{168}{145}(1-x)^{3}\left(1+6 x+16 x^{2}+6 x^{3}+x^{4}\right)
\end{aligned}
$$

We note that the behavior of $D_{1}(x)$ near $x \rightarrow 1$ conforms to the QCD counting rules [22]. This is also the case for $\alpha=1$ in model I of Eq. (6). As we see, at small $x$ 's we can trace a diquark correlation.

The above forms hold by construction at the quark-model scale, where the only degrees of freedom are the valence quarks. To relate to results at experimental scales, the appropriate QCD evolution is necessary [23], which generates radiatively the sea quarks and gluons. The matching prescription based on the requirement that at $\mu=2 \mathrm{GeV}$ the valence quarks carry the experimental momentum fraction of $41.6 \%$ [24] leads to a very low quark model scale, $\mu_{0}=285 \mathrm{MeV}$ (where the valence quarks carry all the momentum). This low value is compatible to the case of the pion in chiral quark models [25,26].

The QCD evolution equations for multi-parton distributions have been derived long ago [6,7]. The DGLAP evolution preserves the GS sum rules [17], hence their satisfaction at the quark-model scale implies satisfaction at higher scales. A simple and practical method of solving the evolution equations numerically is based on the Mellin moments, similarly to the case of sPDFs. One introduces the corresponding moments of the sPDFs and dPDFs,

$$
\begin{aligned}
M_{j}^{n} & =\int_{0}^{1} d x x^{n} D_{j}(x), \\
M_{j_{1} j_{2}}^{n_{1} n_{2}} & =\int_{0}^{1} d x_{1} \int_{0}^{1} d x_{2} \theta\left(1-x_{1}-x_{2}\right) x_{1}^{n_{1}} x_{2}^{n_{2}} D_{j_{1} j_{2}}\left(x_{1}, x_{2}\right),
\end{aligned}
$$

and the moments of the QCD splitting functions

$$
\begin{aligned}
P_{i \rightarrow j}^{n} & =\int_{0}^{1} d x x^{n} P_{i \rightarrow j}(x), \\
P_{i \rightarrow j_{1} j_{2}}^{n_{1} n_{2}} & =\int_{0}^{1} d x x^{n_{1}}(1-x)^{n_{2}} P_{i \rightarrow j_{1} j_{2}}(x), \\
\tilde{P}_{i \rightarrow j_{1} j_{2}}^{n_{1} n_{2}} & =\delta_{j_{1} j_{2}} P_{i \rightarrow j_{1}}^{n_{1}+n_{2}}-\delta_{i j_{1}} P_{j_{1} \rightarrow j_{2}}^{n_{2}}-\delta_{i j_{2}} P_{j_{2} \rightarrow j_{1}}^{n_{1}} .
\end{aligned}
$$

Then the LO dDGLAP evolution equations read $[6,7]$

$$
\frac{d}{d t} M_{j_{1} j_{2}}^{n_{1} n_{2}}=\sum_{i} P_{i \rightarrow j_{1}}^{n_{1}} M_{i j_{2}}^{n_{1} n_{2}}+\sum_{i} P_{i \rightarrow j_{2}}^{n_{2}} M_{j_{1} i}^{n_{1} n_{2}}+\sum_{i}\left(P_{i \rightarrow j_{1} j_{2}}^{n_{1} n_{2}}+\tilde{P}_{i \rightarrow j_{1} j_{2}}^{n_{1} n_{2}}\right) M_{i}^{n_{1}+n_{2}},
$$

where the evolution variable is

$$
t=\frac{1}{2 \pi \beta} \log \left[1+\alpha_{s}(\mu) \beta \log \left(\Lambda_{\mathrm{QCD}} / \mu\right)\right], \quad \beta=\frac{11 N_{c}-2 N_{f}}{12 \pi} .
$$

Partons $i, j_{1}$, and $j_{2}$ may in general represent the valence quarks, the sea quarks, or the gluons, whose distributions are coupled. Moreover, the last term in Eq. (10) couples dPDFs to sPDFs. In this talk we consider only the evolution of the valence quarks which leads to a technical simplification, as in this case there are no partons $i$ decaying into a pair of valence quarks and $P_{i \rightarrow j_{1} j_{2}}=0$. This means that the inhomogeneous term in Eq. (10) vanishes and our evolution equation simply reads

$$
\frac{d}{d t} M_{j_{1} j_{2}}^{n_{1} n_{2}}(t)=\left(P_{j_{1} \rightarrow j_{1}}^{n_{1}}+P_{j_{2} \rightarrow j_{2}}^{n_{2}}\right) M_{j_{1}, j_{2}}^{n_{1} n_{2}}(t)
$$



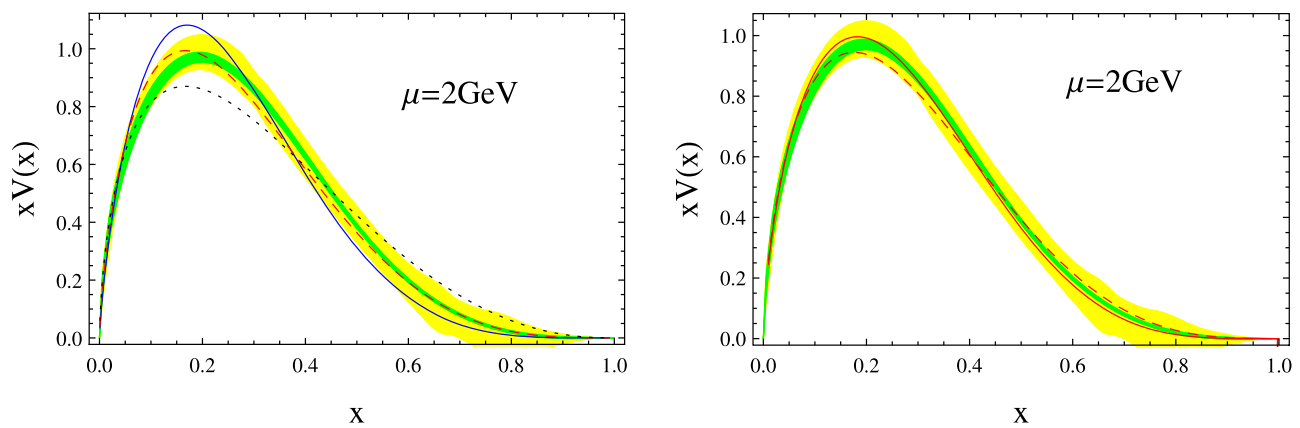

Fig. 1 The valence sPDF of the nucleon (multiplied by $x$ ) at the scale of $\mu=2 \mathrm{GeV}$, plotted as a function of the Bjorken variable $x$. The darker band corresponds to the NNPDF2.3 fit with no LHC data, while the broader light band is the the NNPDF2.3 fit with the collider data only [24]. We show sPDF evolved with the dDGLAP equations from the quark-model scale $\mu_{0}$ to $\mu=2 \mathrm{GeV}$. Left The solid, dashed, and dotted lines correspond to the valon model $|\psi(x)|^{2}=A x^{\alpha}$ with $\alpha=1,1 / 2$, and 0 , respectively. Right The solid and dashed lines correspond to the model $|\psi(x)|^{2}=A(1-x)^{a}$ with $a=2$ and $a=1$, respectively

Recall that for sPDFs the corresponding evolution equation is

$$
\frac{d}{d t} M_{j}^{n}(t)=P_{j \rightarrow j}^{n} M_{j}^{n}(t) .
$$

Note that due to the presence of correlations we have $M_{j_{1} j_{2}}^{n_{1} n_{2}}(t) \neq M_{j_{1}}^{n_{1}}(t) M_{j_{2}}^{n_{2}}(t)$ and the system is not separable. The solutions of Eqs. $(11,12)$ are

$$
\begin{aligned}
M_{j}^{n}(t) & =e^{P_{j \rightarrow j}^{n}\left(t-t_{0}\right)} M_{j}^{n}\left(t_{0}\right), \\
M_{j_{1} j_{2}}^{n_{1} n_{2}}(t) & =e^{\left(P_{j_{1} \rightarrow j_{1}}^{n_{1}}+P_{j_{2} \rightarrow j_{2}}^{n_{2}}\right)\left(t-t_{0}\right)} M_{j_{1} j_{2}}^{n_{1} n_{2}}\left(t_{0}\right) .
\end{aligned}
$$

The inverse Mellin transform brings us to the evolved solution in the $x$-space, namely

$$
\begin{aligned}
D_{j}(x ; t) & =\int_{C} \frac{d n}{2 \pi i} x^{-n-1} M_{j}^{n}(t), \\
D_{j_{1} j_{2}}\left(x_{1}, x_{2} ; t\right) & =\int_{C} \frac{d n_{1}}{2 \pi i} x_{1}^{-n_{1}-1} \int_{C^{\prime}} \frac{d n_{2}}{2 \pi i} x_{2}^{-n_{2}-1} M_{j_{1}, j_{2}}^{n_{1}, n_{2}}(t),
\end{aligned}
$$

where $n$ and $n^{\prime}$ are treated as complex variables and the contours $C$ and $C^{\prime}$, lying right to all singularities of $M$, are bended by $45^{\circ}$ (see e.g. Ref. [27]). The bending of the contours helps with the pace of numerical convergence. Schwarz's reflection principle $M_{n^{*}}=M_{n}^{*}$ is satisfied since the anomalous dimensions are real for any integer $n$ and thus one segment of the bended contour is needed in the evaluation. The method is most practical when the moments $M$ are analytic functions of their arguments; if they were not (as could be the case when some numerical fits to the data are made), then they should be approximated with analytic functions, e.g., with sums of the Euler Beta functions, whence the procedure may be carried out with no difficulty.

Next, we proceed to presenting our results of the evolution in the considered model. Before showing the main findings for the valence dPDF, we explore the valence sPDF, where the results can be straightforwardly compared to the existing data parameterizations. Figure 1 presents the valence quark distribution, customarily multiplied by $x$, evolved to the benchmark scale of $\mu=2 \mathrm{GeV}$ and compared to the NNPDF2.3 fits [24], indicated with bands (the darker narrow band is the fit excluding the LHC data, and the broad band uses the collider data only). The reasonable reproduction of the valence sPDF gives us confidence that the simple model of Eqs. $(3,4)$ grasps the most essential features of the valence parton distributions of the nucleon, particularly for the case $a=2, \psi(x)=\sqrt{3}(1-x)$. We may thus move towards the double distributions. The effect of the evolution on the valence dPDFs is presented in Fig. 2, where we show the contour maps of $x_{1} x_{2} D_{2}\left(x_{1}, x_{2}\right)$ at three scales: the quark-model scale $\mu_{0}$, and the higher scales $\mu=2 \mathrm{GeV}$ and $\mu=1 \mathrm{TeV}$. Of course, the support of the function is the region $x_{1}+x_{2} \leq 1, x_{i} \geq 0$. We observe how the dDGLAP equations cause the drift of the distributions towards low values of $x_{1}$ and $x_{2}$. This drift to low values of $x$ is well-known for the case of the DGLAP evolution of sPDFs; it has also been observed for the dPDFs [17]. We note the lack 

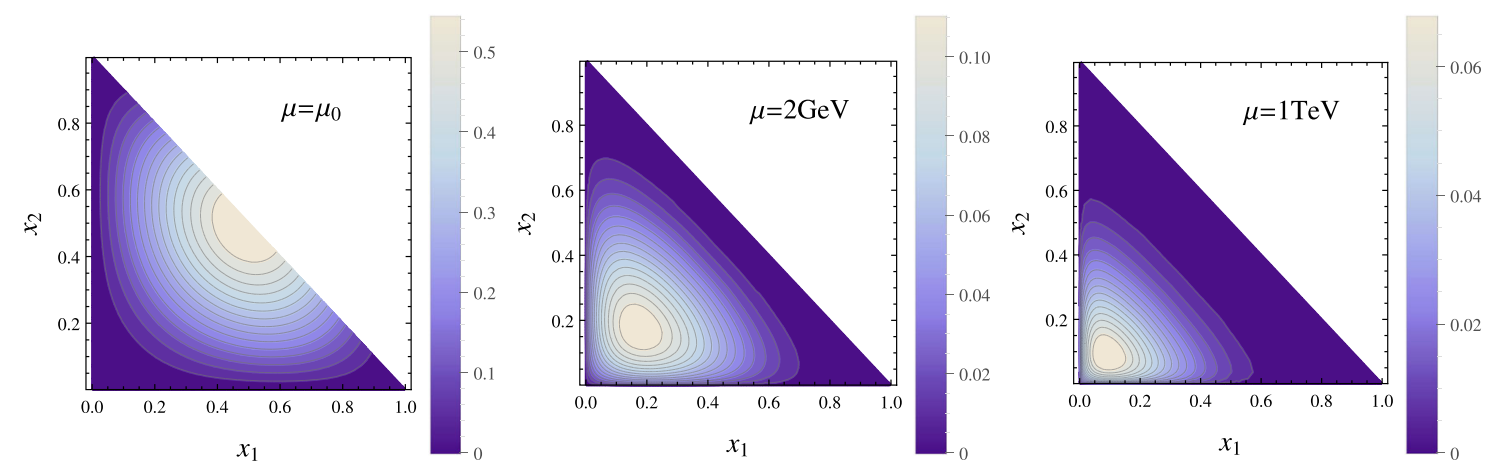

Fig. 2 Contour plots of the valence dPDF of the nucleon multiplied by $x_{1} x_{2}$, i.e., the quantity $x_{1} x_{2} D_{2}\left(x_{1}, x_{2}\right)$, at the scale $\mu_{0}$ and evolved to $\mu=2 \mathrm{GeV}$ and $\mu=1 \mathrm{TeV}$ with the LO dDGLAP equations. Model with $a=2$ corresponding to $\psi(x)=\sqrt{3}(1-x)$
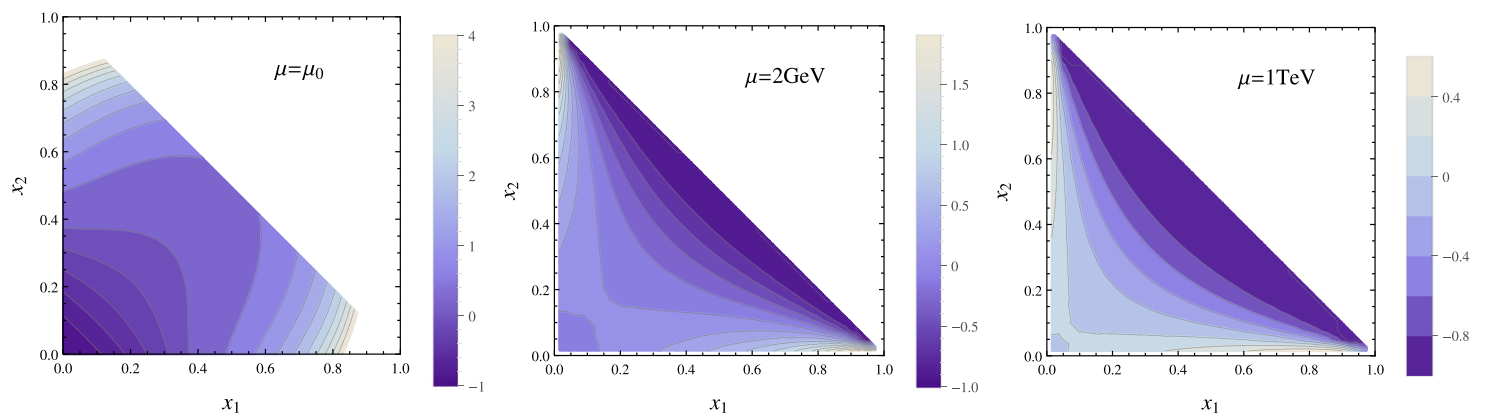

Fig. 3 Contour plots of the valence quark correlation function $\rho\left(x_{1}, x_{2}\right)=D_{2}\left(x_{1}, x_{2}\right) /\left[D_{1}\left(x_{1}\right) D_{1}\left(x_{2}\right)\right]-1$, at the scales $\mu_{0}$, $\mu=2 \mathrm{GeV}$, and $\mu=1 \mathrm{TeV}$. Model with $a=2$ corresponding to $\psi(x)=\sqrt{3}(1-x)$

of factorization in the $x_{1}$ and $x_{2}$ variables within the DGLAP approach, as emphasized in Ref. [28,29]. The behavior at the end-lines [30] shows that for a fast variable and a slow variable, i.e., $x_{1} \rightarrow 1$ and $x_{2} \rightarrow 0$, the correlation becomes small, since

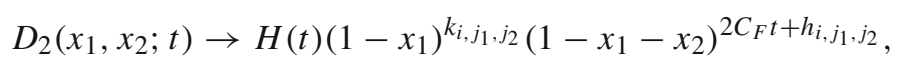

a feature confirmed with our numerical analysis.

For a probability interpretation to hold, it is necessary that the evolution equations preserve the positivity of the initial condition. Amusingly, it has been found that the upward evolution for SPDFs produces positive distributions [31], unlike the downward evolution where a lower bound for the evolution ratio is found on this basis [27] (cf. also the irreversible evolution of Ref. [32]). Within the considered model, the upward evolution preserves the positivity of the valence dPDF, as can be seen from Fig. 2.

In Fig. 3 we plot the double-parton correlation function as defined in Eq. (1). As the scale increases, the correlation becomes large and positive along the lines $x_{i}=0$, while in the central region it is negative. At the line of the kinematic constraint $x_{1}+x_{2}=1$ we find $\rho\left(x_{1}, x_{2}\right)=-1$. The results of Fig. 3 show that the valence dPDF cannot be approximated with the uncorrelated product of the SPDFs. We note that our plots bare qualitative similarity to the results obtained in the MIT bag model [15].

We end with some remarks concerning the longitudinal and transverse degrees of freedom. Our calculation is essentially 1-dimensional, as the transverse dynamics plays no active role. This dimensional reduction could be possible if the relative momentum between the two quarks is assumed to be small. To analyze this assumption from a non-perturbative point of view, let us remind ourselves that the transverse lattice approach [33] allows to effectively freeze the transverse degrees of freedom when the transverse spacing, $\mathbf{a}_{\perp}$, becomes larger than the characteristic resolution of the parton momentum in the hadron, $\sim 1 / \Lambda_{\mathrm{QCD}}$. This also limits the multiparton Fock state by powers of $a_{\perp}$. For instance, for the nucleon $N=q^{3}+a_{\perp}^{3} q^{4} \bar{q}+a_{\perp}^{4} q^{3} G+\cdots$. However, as soon as $a_{\perp}$ starts decreasing, gluons and sea quarks are radiated and it is amazing that the DGLAP evolution equations roughly reproduce the observed behavior for SPDF [26]. 
A simple way to mimic the transverse lattice dynamics is by using a 2-dimensional harmonic oscillator to trap the partons in the hadron [34] in the transverse plane, with a mass squared operator given by

$$
M^{2}=\sum_{i}\left[\frac{\mathbf{k}_{i \perp}^{2}+m_{i}^{2}}{x_{i}}+\sigma^{2} x_{i} \mathbf{b}_{i}^{2}\right]
$$

Here, $x_{i}$ are the longitudinal momentum fractions fulfilling $\sum_{i} x_{i}=1, \mathbf{b}_{i}$ are the impact-parameter variables, $\mathbf{k}_{\perp, i}$ their corresponding Fourier-conjugate variables, and $\sigma$ is the oscillator constant. If $\psi_{n_{\perp}}(x, \mathbf{b})$ is the single particle two dimensional $\mathrm{HO}$ wave function normalized as $\int d^{2} b\left|\psi_{n_{\perp}}(x, \mathbf{b})\right|^{2}=1$ with quantum numbers $\mathbf{n}_{\perp}=\left(n_{x}, n_{y}\right)$ and mass squared $M^{2}=2 \sigma\left(n_{x}+n_{y}+1\right)$, then the general solution is a symmetrized product of factorized states

$$
\Psi_{\mathbf{n}_{\perp}}\left(x_{1}, \mathbf{b}_{1}, \ldots, x_{N}, \mathbf{b}_{N}\right)=\varphi\left(x_{1}, \ldots, x_{N}\right) \prod_{i=1}^{N} \psi_{n_{i, \perp}}\left(x_{i}, \mathbf{b}_{i}\right) \chi_{\text {spin-flavor }} .
$$

Due to the fact that the single-particle wave functions have an $x$-independent normalization, one clearly has

$$
D_{N}\left(x_{1}, \ldots, x_{N}\right)=\prod_{i} \int d^{2} b_{i}\left|\Psi_{\mathbf{n}_{\perp}}\left(x_{1}, \mathbf{b}_{1}, \ldots, x_{N}, \mathbf{b}_{N}\right)\right|^{2}=\left|\varphi\left(x_{1}, \ldots, x_{N}\right)\right|^{2} .
$$

Note that here the variables $x_{i}$ are fixed, i.e., they are not dynamical, and we can still multiply by an arbitrary function $\varphi\left(x_{1}, \ldots, x_{N}\right)$ which would be generated after requantization by some unspecified 1-dimensional dynamics, say $M^{2}=M_{\mathrm{HO}}^{2}+M_{1+1}^{2}$. Our model in Eq. (2) corresponds to the product $\varphi\left(x_{1}, \ldots x_{N}\right)=$ $\prod_{i} \psi\left(x_{i}\right)$. This is similar to the Born-Oppenheimer approximation where the longitudinal variables obey classical dynamics. In this extreme case the mass terms give after minimization $x_{i}=m_{i} / m$, with $m=$ $\sum_{i} m_{i}$ yielding a contribution $m^{2}$ to $M^{2}$ and $D_{N}\left(x_{1}, \ldots, x_{N}\right) \rightarrow \prod_{i} \delta\left(x_{i}-m_{i} / m\right)$, which for equal masses corresponds to locating the distribution in our Eq. (2) at the maximum, $x_{i}=1 / N$. That model breaks the transverse translational invariance, which can be restored by projecting onto zero total transverse momentum using the Peierls-Yoccoz method [18], such that for $m_{i}=0$ one finds $M_{N}^{2}=2 N \sigma$ for the symmetric state. A straightforward calculation with the rest frame wave function yields the following result for the unintegrated dPDF:

$$
D_{2}\left(x_{1}, x_{2}, \mathbf{k}_{\perp}\right)=\int d^{2} b d^{2} B\left|\Psi_{0}\left(x_{1}, \mathbf{B}+\frac{1}{2} \mathbf{b}, x_{2}, \mathbf{B}-\frac{1}{2} \mathbf{b}\right)\right|^{2} e^{i \mathbf{b} \cdot \mathbf{k}_{\perp}}=D_{2}\left(x_{1}, x_{2}\right) e^{-\frac{\mathbf{k}_{\perp}^{2}}{4 \sigma} \frac{x_{1}+x_{2}}{x_{1} x_{2}}} .
$$

Thus, there exists a transverse dynamics given by Eq. (18) where the effective 1-dimensional treatment used here holds, but it does not support the often assumed transverse-longitudinal factorization.

Open Access This article is distributed under the terms of the Creative Commons Attribution License which permits any use, distribution, and reproduction in any medium, provided the original author(s) and the source are credited.

\section{References}

1. Bartalini, P., Berger, E., Blok, B., Calucci, G., Corke, R., et al.: Multi-parton interactions at the LHC. arXiv:1111.0469 (2011)

2. Snigirev, A.M.: Double-parton distributions in QCD. Phys. At. Nucl. 74, 158-165 (2011)

3. d'Enterria, D., Snigirev, A.M.: Same-sign WW production in proton-nucleus collisions at the LHC as a signal for double parton scattering. Phys. Lett. B 718, 1395-1400 (2013)

4. Luszczak, M., Maciula, R., Szczurek, A.: Production of two $c \bar{c}$ pairs in double-parton. Phys. Rev. D 85, 094034 (2012)

5. Kuti, J., Weisskopf, V.F.: Inelastic lepton - nucleon scattering and lepton pair production in the relativistic quark parton model. Phys. Rev. D 4, 3418-3439 (1971)

6. Kirschner, R.: Generalized Lipatov-Altarelli-Parisi equations and jet calculus rules. Phys. Lett. B 84, 266 (1979)

7. Shelest, V., Snigirev, A., Zinovev, G.: The multiparton distribution equations in QCD. Phys. Lett. B 113, 325 (1982)

8. Konishi, K., Ukawa, A., Veneziano, G.: Jet calculus: a simple algorithm for resolving QCD jets. Nucl. Phys. B 157, 45107 (1979)

9. Collins, J.C., Soper, D.E.: Back-to-back jets in QCD. Nucl. Phys. B 193, 381 (1981) 
10. Diehl, M.: Multiple interactions and generalized parton distributions. PoS DIS2010, p. 223 (2010)

11. Diehl, M., Schafer, A.: Theoretical considerations on multiparton interactions in QCD. Phys. Lett. B 698, 389-402 (2011)

12. Diehl, M., Ostermeier, D., Schafer, A.: Elements of a theory for multiparton interactions in QCD. JHEP 1203, 089 (2012)

13. Blok, B., Dokshitzer, Y., Frankfurt, L., Strikman, M.: The four jet production at LHC and tevatron in QCD. Phys. Rev. D 83, $071501(2011)$

14. Diehl, M., Kasemets, T.: Positivity bounds on double parton distributions. JHEP 1305, 150 (2013)

15. Chang, H.-M., Manohar, A.V., Waalewijn, W.J.: Double parton correlations in the bag model. Phys. Rev. D 87, 034009 (2013)

16. Rinaldi, M., Scopetta, S., Vento, V.: Double parton correlations in constituent quark models. Phys. Rev. D 87, 114021 (2013)

17. Gaunt, J.R., Stirling, W.J.: Double parton distributions incorporating perturbative QCD evolution and momentum and quark number sum rules. JHEP 1003, 005 (2010)

18. Betz, M., Goldflam, R.: Boosting the bag. Phys. Rev. D 28, 2848 (1983)

19. Ruiz Arriola, E.: In: Proceedings of the Mini-Workshop. Hadrons as Solitons, Bled (Slovenia), 9-17 July 1999, pp. 5-16 (1999). hep-ph/9910382

20. Hwa, R.C., Zahir, M.S.: Parton and valon distributions in the nucleon. Phys. Rev. D 23, 2539 (1981)

21. Hwa, R.C., Yang, C.B.: Parton distributions in the valon model. Phys. Rev. C 66, 025204 (2002)

22. Lepage, G.P., Brodsky, S.J.: Exclusive processes in perturbative quantum chromodynamics. Phys. Rev. D 22, 2157 (1980)

23. Broniowski, W., Ruiz Arriola, E., Golec-Biernat, K.: Generalized parton distributions of the pion. AIP Conf. Proc. 1030, 286290 (2008)

24. Ball, R.D., Bertone, V., Carrazza, S., Deans, C.S., Del Debbio, L. et al.: Parton distributions with LHC data. Nucl. Phys. B 867, 244-289 (2013)

25. Davidson, R., Ruiz Arriola, E.: Structure functions of pseudoscalar mesons in the SU(3) NJL model. Phys. Lett. B 348, 163169 (1995)

26. Broniowski, W., Ruiz Arriola, E., Golec-Biernat, K.: Generalized parton distributions of the pion in chiral quark models and their QCD evolution. Phys. Rev. D 77, 034023 (2008)

27. Ruiz Arriola, E.: NLO evolution for large scale distances, positivity constraints and the low-energy model of the nucleon. Nucl. Phys. A 641, 461-475 (1998)

28. Snigirev, A.M.: Double parton distributions in the leading logarithm approximation of perturbative QCD. Phys. Rev. D 68, $114012(2003)$

29. Korotkikh, V.L., Snigirev, A.M.: Double parton correlations versus factorized distributions. Phys. Lett. B 594, 171-176(2004)

30. Snigirev, A.M.: Asymptotic behavior of double parton distribution functions. Phys. Rev. D 83, 034028 (2011)

31. Llewellyn Smith, C.H., Wolfram, S.: Positivity constraints on quark and gluon distributions in QCD. Nucl. Phys. B 138, $333(1978)$

32. Teryaev, O.V.: The irreversibility of QCD evolution equations. Phys. Part. Nucl. 36, S160-S163 (2005)

33. Burkardt, M., Dalley, S.: The relativistic bound state problem in QCD: transverse lattice methods. Prog. Part. Nucl. Phys. 48, 317-362 (2002)

34. Vary, J.P., Honkanen, H., Li, J., Maris, P., Brodsky, S.J. et al.: Hamiltonian light-front field theory in a basis function approach. Phys. Rev. C 81, 035205 (2010) 